\title{
Correspondence
}

Arch. Dis. Childh., 1969, 44, 784.

\section{Intelligence and Retinoblastoma}

Sirs,

Miss M. Williams' paper in the April 1968 issue of your journal (p. 204) records evidence from control material that children blind from retinoblastoma have superior intelligence. The control material consisted of two series, one being a group of children blinded from causes other than retinoblastoma and the other a group of sighted children from ordinary schools. Both these control groups seem to have anomalous features.

The children blinded from causes other than retinoblastoma were a heterogeneous group. More than a third of the 74 cases had some useful vision (counting fingers to 6/60) and a further unstated number had perception of light, while 45 out of the 50 retinoblastoma children were totally blind. Almost a third of the control group were cases of retrolental fibroplasiaan affliction of markedly premature children often grossly affected mentally. The fact that these particular children attended a school for blind children not suffering from multiple handicaps does mean in this context that they were not educationally subnormal. It is unfortunate that such a high proportion of children at risk mentally should be included in the control material. On visual and mental criteria, the 74 nonretinoblastoma children are hardly a convincing control series.

The findings on the control material of sighted normal children should give incontrovertible conclusions. One is, however, puzzled by the fact that this group is shown to rate in intelligence no higher than the group of non-retinoblastoma children with their heavy proportion of cases of retrolental fibroplasia. The question arises whether the Williams test used, one designed for visually handicapped children, does not put sighted children at a disadvantage. This is indeed a point raised by Miss Williams and dismissed as of no consequence, but the findings are none-the-less puzzling.

Until there is better evidence than is as yet available, most ophthalmologists would agree with the considered opinion of Dr. R. Forrester whom Miss Williams quotes. He gives succinctly the essential aspects of the question at issue and will bear repetition:

'Of all the causes of blindness in young children the retinoblastoma is the one in which the child is allowed to avoid: (a) premature birth and its subsequent handicaps; (b) brain damage and its associated neurological disorders-the disorder is confined to the eye; (c) many of these children did have some sight for months or even a year or more. This may just have been sufficient to lift them over the impenetrable barrier which other congenitally blind children cannot surmount. The scales are weighted in favour of the retinoblastoma child just as they are weighted against the average child with other forms of congenital blindness; I think this explains the difference.'
One further point may perhaps be made. In the context of educational achievement, the child blind from retinoblastoma has a double advantage; not only did he have good vision before becoming blind, but his blindness when it came-generally quickly - was total and so excluded delay in registration as blind and other halfmeasures.

19 Parham Court, Grand Avenue, Worthing,

Sussex.

We showed Professor Sorsby's letter to Miss M. Williams, who commented as follows:

The question (paragraph 3 of Professor Sorsby's letter) whether the Williams Test puts sighted children at a disadvantage was answered by the research datathe fact that an unselected sighted sample gained a mean IQ on the Williams Test of just over 100 . (In confirmation of this, a recent German study showed a close correspondence between the scores of sighted children on the German edition of the Williams Test and their scores on the German translation of the Terman-Merrill Scale.) Thus it follows that the non-retinoblastoma blind control-despite the expectation that it might be biased by the presence of retrolental fibroplasia children-was not so biased; it had the same average intelligence as an unselected group of sighted children. Therefore, the difference between the mean IQ of the retinoblastoma sample and that of the non-retinoblastoma sample is not capable of explanation solely on Dr. Forrester's quoted hypothesis; they differed (i.e. the retinoblastoma children) by about 17 points of IQ from a non-retinoblastoma sample, from an unselected sighted sample, and from the established norm of the Williams Test (derived from a blind school sample of considerable size).

One is puzzled by this result, but the evidence does not suggest any inferiority in the scores of the control groups-IQ 100 is average. It does indicate clearly the superiority of the retinoblastoma scores.

The number of controls who had perception of light was not specified as there would have been little relevance in stressing this. Any appreciable proportion of cases with perception of light in the non-retinoblastoma blind sample would have presumably given them an advantage over the almost entirely totally blind retinoblastoma group.

School of Education,

M. WILLIAMS

University of Birmingham. 